\title{
Numerical Investigation of Bubble Induced Marangoni Convection: Some Aspects of Bubble Geometry
}

\author{
Séamus M. O’Shaughnessy, Anthony J. Robinson* \\ Department of Mechanical \& Manufacturing Engineering, Parsons Building, Trinity College \\ Dublin, Ireland \\ +3531 8963919
}

arobins@tcd.ie

http://www.mme.tcd.ie/research/heat transfer.php

Thermocapillary or Marangoni convection is a surface tension driven flow that occurs when a gas-liquid or vapor-liquid interface is subjected to a temperature gradient. In the past, the contribution to local heat transfer arising from Marangoni convection has been overlooked as insignificant since under earth gravity it is overshadowed by buoyant convection. This study numerically investigates some aspects of bubble size and shape on local wall heat transfer resulting from Marangoni convection about individual bubbles on a heated wall immersed in a liquid silicone oil layer $(\operatorname{Pr}=110)$ of depth $5 \mathrm{~mm}$. It was found that increasing bubble volume causes an increase in the area over which Marangoni convection has affect. Heat transfer therefore increases with bubble size. The surface averaged hot wall heat transfer is not affected greatly by bubble shape. The surface averaged heat transfer over the effective area on both the hot and cold walls is affected dramatically by bubble size, but the increase is more profound on the cold wall.

Marangoni, thermocapillary, bubble, microgravity, heat transfer enhancement

\section{Introduction}

Larkin (1970) was possibly the first to numerically investigate Marangoni convection caused by the presence of a bubble situated on a heated wall, specifically looking at the contribution to local heat transfer. Larkin varied the Prandtl and Marangoni numbers to obtain transient solutions for the thermal and flow fields. The influence of thermocapillarity on the Nusselt number was investigated, with Larkin reporting that above a Marangoni number of $10^{5}$, an increase in the rate of heat transfer of $30 \%$ was achievable. Larkin therefore concluded that until this Marangoni number was reached, Marangoni convection did not play an important role in heat transfer. Arlabosse et al. (2000) analyzed the influence of the Marangoni, Prandtl, and Bond numbers on the interfacial velocity profiles. The enhancement in heat flux due to thermocapillarity was measured in the vicinity of the bubble. For their experiments Arlabosse 
et al. concluded that beyond two bubble diameters heat transfer is dominated by conduction. It was determined that the ratio between heat transfer with an air bubble to that of pure conduction changes with the square root of the Marangoni number.

In parallel with their experiments, Betz and Straub (2001) performed numerical simulations of bubble induced Marangoni convection using a 2D mathematical model employing a finite element method. Solutions were obtained at both terrestrial and microgravity conditions as a means of predicting the substantial differences in heat transfer and fluid flow on earth and in microgravity. Modeling of the problem was performed in the range $7 \leq \operatorname{Pr} \leq 120$ and $1000 \leq \mathrm{Ma} \leq 27800$. It was found that the numerical results for Nusselt number produced by a 'real' shaped bubble were greater than those determined experimentally. Betz and Straub attributed this to the fact that the 2D model could not accurately depict the 3D scenario. The enhancement of heat transfer was expressed as a Nusselt number, defined as the ratio of heat transfer due to thermocapillary convection around the bubble in relation to heat conduction in the liquid about an adiabatic bubble, or equivalently

$$
N u=q_{M a}^{\prime \prime} / q_{c o n d, A B}^{\prime \prime}
$$

The Prandtl number for this study was $\operatorname{Pr}=110$. Betz and Straub (2001) produced results where several experimental studies were compared with numerical data which indicated a relative independence of Marangoni flow to $\operatorname{Pr}$ number. An inverse relationship between velocity and $\operatorname{Pr}$ number was proposed by Arlabosse et al. (1999), but in their numerical study, simulations were carried out at fixed Rayleigh numbers, as their dimensionless approach allowed for the decoupling of $R a$ and $M a$. In reality this is impossible, since any change in viscosity or thermal diffusivity will change $\operatorname{Pr}, M a$ and $R a$. Silicone oils are primarily used to study thermocapillary convection. Since the temperature derivative of surface tension is quite similar for many silicone oils of different viscosity, lower $\mathrm{Pr}$ oils will have greater $\mathrm{Ma}$ numbers for the same operating conditions, and hence greater enhancement of heat transfer.

With regard to the application of thermocapillary convection to boiling scenarios, Barthes et al. (2007) studied the influence of non-condensable gas influence on Marangoni convection during single vapor bubble growth in a subcooled liquid. More recently, Raj and Kim (2007) presented their numerical investigation of thermocapillary convection in reduced gravity environments, entailing a qualitative study on the effects of dissolved gas content, bubble 
shape and size, and heat transfer coefficient on the strength of thermocapillary convection. Steady-state simulations were carried out to calculate the flow around bubbles of various sizes with different amounts of dissolved gas for subcooled boiling in reduced gravity. For a given heat transfer coefficient with increasing bubble size, it was found that the strength of the thermocapillary convection increased before peaking and then decreasing. Furthermore the peak was observed to occur at lower diameters when increasing the heat transfer coefficient. It was determined that an increase in dissolved gas content promotes the interfacial temperature gradient required for thermocapillary convection, but the simultaneous increase in bubble size reduced the gradient along the interface. Therefore, Raj and Kim concluded that the strength of thermocapillary convection is an indirect function of dissolved gas content, which in turn influences variation in heat transfer coefficient, and consequently the bubble shape and size.

There remains however limited information regarding the contribution to heat transfer resulting from bubble induced Marangoni convection. This study aims to elucidate the effect of bubble size and shape on the local wall heat transfer on both the heated and cooled surfaces in microgravity.

\section{Numerical Formulation}

For thermocapillary convection around a bubble of radius $\mathrm{R}_{\mathrm{b}}$, within a channel of height $\mathrm{H}$, under zero-gravity conditions, the mass and heat transport mechanisms are characterized by the Prandtl and Marangoni numbers, defined respectively as (Arlabosse et al., 1999; Reynard et al., 2005),

$$
\begin{gathered}
\operatorname{Pr}=\frac{v}{\alpha} \\
M a=-\left(\frac{\partial \sigma}{\partial T}\right) \times \frac{\Delta T}{\mu \alpha} \times \frac{R_{b}{ }^{2}}{H}
\end{gathered}
$$

A two-dimensional axisymmetric model of the problem was formulated using the commercial software package FLUENT® version 6.2.16. The governing equations for the problem are continuity, momentum and energy, each in steady-state. For incompressible flow with constant physical properties,

$$
\nabla \cdot \vec{v}=0
$$




$$
\begin{aligned}
& \rho(\vec{v} \cdot \nabla \vec{v})=-\nabla p+\mu \nabla^{2} \vec{v} \\
& \rho C_{p}(\vec{v} \cdot \nabla T)=k \nabla^{2} T
\end{aligned}
$$

The model domain is shown in Figure 1. In accordance with previous numerical investigations on Marangoni convection (Larkin, 1970; Arlabosse et al. 1999), the following assumptions are made in the analysis:

- Motion is 2D axisymmetric in cylindrical coordinates

- Heat flux is zero at the bubble interface

- Incompressibility of the liquid

- Constant physical properties

- Gravitational acceleration is zero

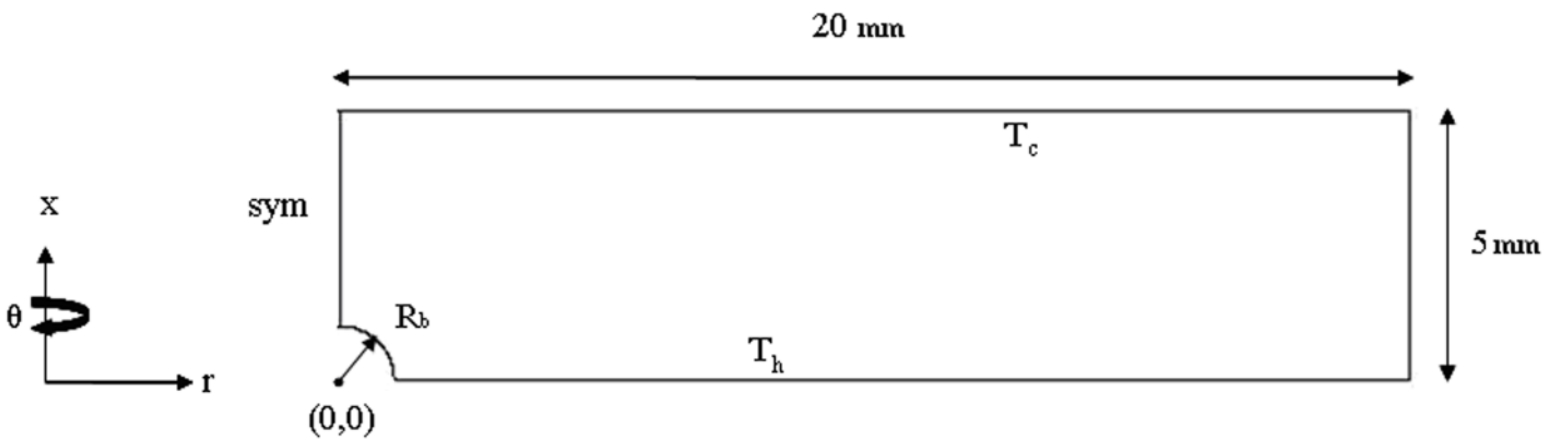

Figure 1: Numerical domain

The vertical wall denoted 'sym' signifies the axis of symmetry. Two horizontal walls are separated by a distance $H$ equal to $5 \mathrm{~mm}$. The upper horizontal wall is no slip, constant temperature;

$$
\vec{v}=0, \quad T=T_{c}=300 K
$$

The lower horizontal wall is also no slip, constant temperature;

$$
\vec{v}=0, \quad T=T_{h}=350 \mathrm{~K}
$$

The vertical wall placed twenty millimeters from the centre of the bubble has a no-slip, adiabatic condition.

$$
\vec{v}=0, \quad \vec{n} \cdot(k \nabla T)=0
$$

The bubble is represented by a boundary upon which a Marangoni stress is directly applied. 


$$
\vec{\tau}=\frac{d \sigma}{d T} \nabla T, \quad \vec{n} \cdot(k \nabla T)=0
$$

Simulations were conducted with hemispherical bubbles of radius $0.5 \mathrm{~mm}, 1 \mathrm{~mm}, 1.5 \mathrm{~mm}$ and $2 \mathrm{~mm}$, truncated $1 \mathrm{~mm}$ radius hemispherical bubbles with contact angles of $66^{\circ}$ and $45^{\circ}$ respectively, and an elliptical bubble with the same volume as the $2 \mathrm{~mm}$ radius hemispherical bubble. Some of the bubble shapes are shown in Figure 2.

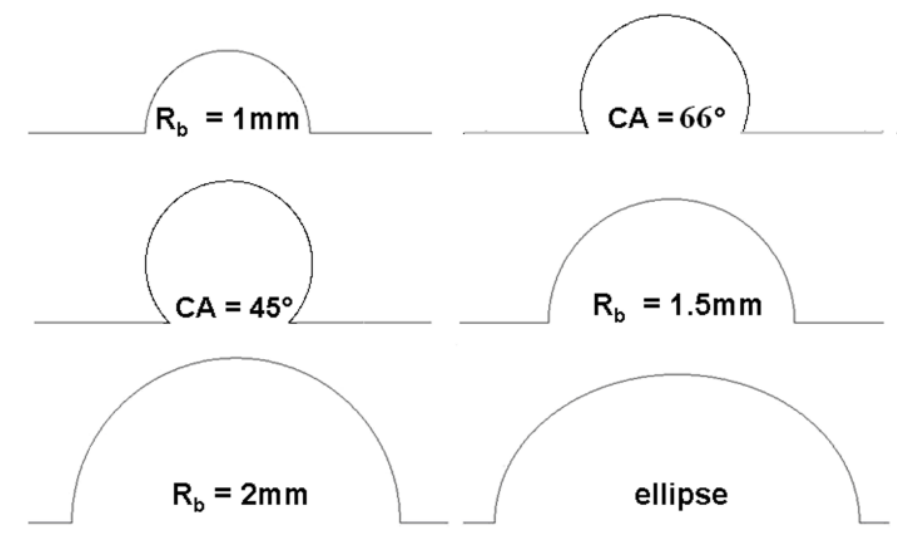

Figure 2: Some bubble shapes simulated

Grid independence was achieved by increasing the number of structurally arranged quadrilateral cells from 23,000 to 86,000 and plotting the convergence of certain parameters of interest such as free surface velocity, and also tracking global parameters such as total rate of heat transfer through the system. To validate the numerical procedure, results were compared with experimental data provided by Arlabosse et al. (2000). The agreement between the experimentally measured non-dimensional interface velocities and the simulation is quite good. The validation simulations are described in further detail in O'Shaughnessy and Robinson (2008). Arlabosse et al. also quantified the increase in the average heat flux across a heat flux sensor situated on the opposite wall to that of the bubble. Comparison between their experimental data and the numerically simulated data was found to be acceptable.

\section{Results}

Figure 3 shows the temperature contours and velocity streamlines for the $1 \mathrm{~mm}$ radius hemispherical bubble. Since the temperature difference was kept constant for all simulations, the Marangoni number was modified by changing the bubble geometry as per Eq 3 . The figure shows the temperature contours which are a feature of thermocapillary convection. In 
the absence of the bubble, the contours form parallel horizontal lines; a situation characteristic of pure molecular conduction. Although not evident in Figure 3, this also occurs far from the bubble. In the vicinity of the bubble, the temperature contours are curved towards the heated wall by the flow of cooler liquid toward the hot wall. The liquid jet, by flowing outward along the centerline of the geometry, causes the temperature contours to project outward. The proximity of the upper wall, which has a no-slip condition, limits the extent the jet can travel in this geometry. From Figure 3, it is evident that the thermocapillary flow field increases heat transfer by removing heat from the wall in the vicinity of the bubble and transporting it into the bulk region above the bubble.

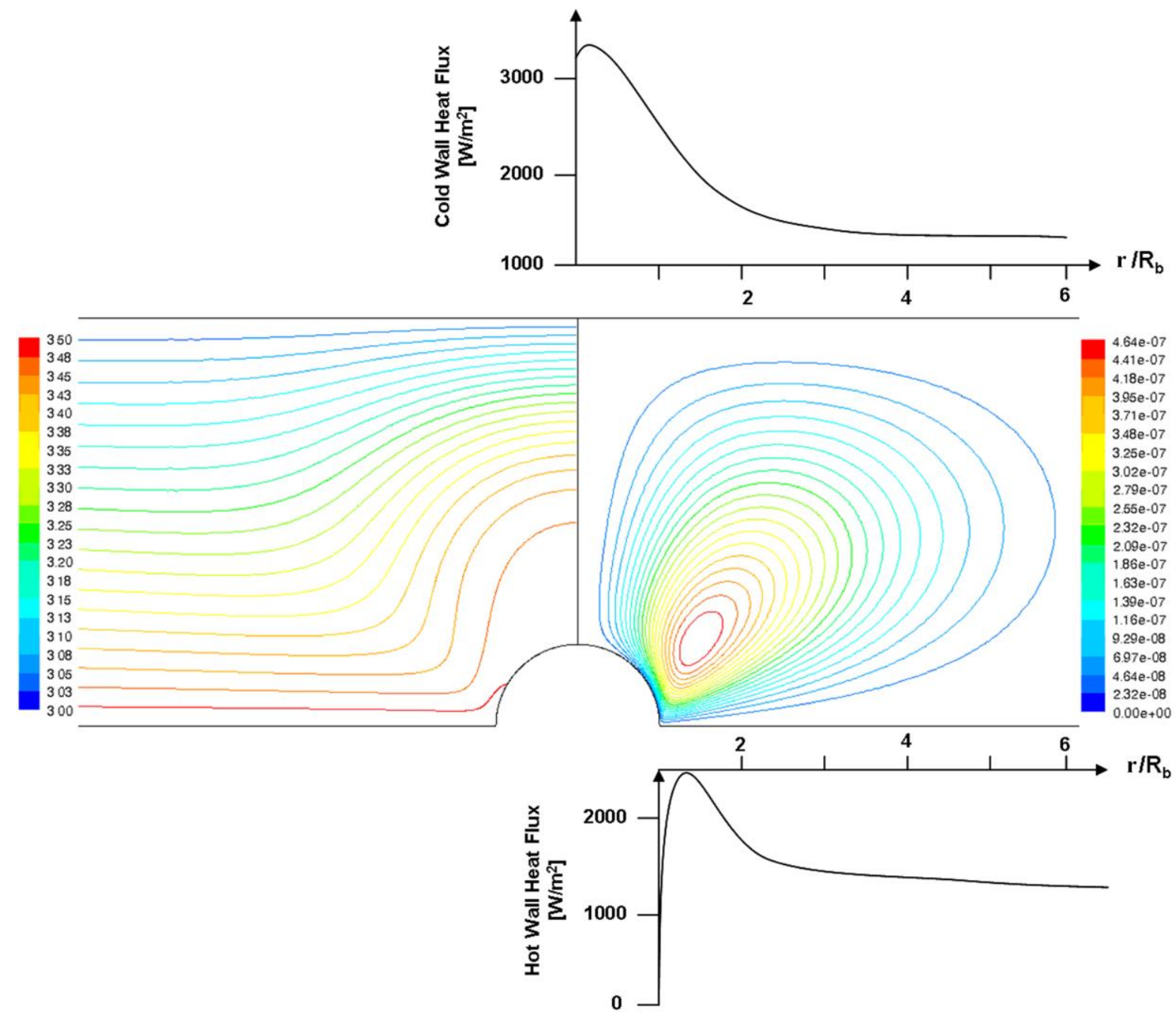

Figure 3: Temperature contours/velocity streamlines for the $1 \mathrm{~mm}$ radius hemispherical bubble

The Marangoni convection induced by the bubble causes a region of enhanced heat transfer in the vicinity of the bubble. At some distance from the bubble, thermocapillary convection will cease to augment local wall heat transfer. This location can be calculated by analyzing 
the heat flux distribution along the hot and cold walls. Initially, simulations were carried out over a similar domain in the absence of the bubble to predict the heat transfer due to pure molecular diffusion and establish a 'base-line' to which subsequent conditions can be compared. Consistent with analytical predictions, the heat flux along the channel walls was constant in the absence of the bubble, and is hence denoted $q^{\prime \prime}$ cond. The criterion for enhancement is defined such that heat flux magnitudes are recorded until the value of wall heat flux falls below $1.05 \times q^{\prime \prime}$ cond. The radial location where heat flux values fall to within $5 \%$ of $q^{\prime \prime}$ cond is termed the effective radius, $R_{\text {eff }}$, and is measured from the centre of the bubble (see Eq 11). The effective area is then calculated based on $R_{\text {eff }}$ (see Eq 12). On the hot wall, the effective area is measured radially outward from the triple contact point (wall-vaporliquid interface). For the cold wall, the effective area is measured radially outward from the axis of symmetry, as depicted in Figure 3 previously.

$$
\begin{array}{r}
R_{e f f}=\left.r\right|_{q^{\prime \prime}=1.05 \times q_{\text {oond }}^{0}} \\
A_{e f f, h w}=\pi\left(R_{e f f}^{2}-r_{\text {tcp }}^{2}\right), \quad A_{e f f, c w}=\pi R_{e f f}^{2}
\end{array}
$$

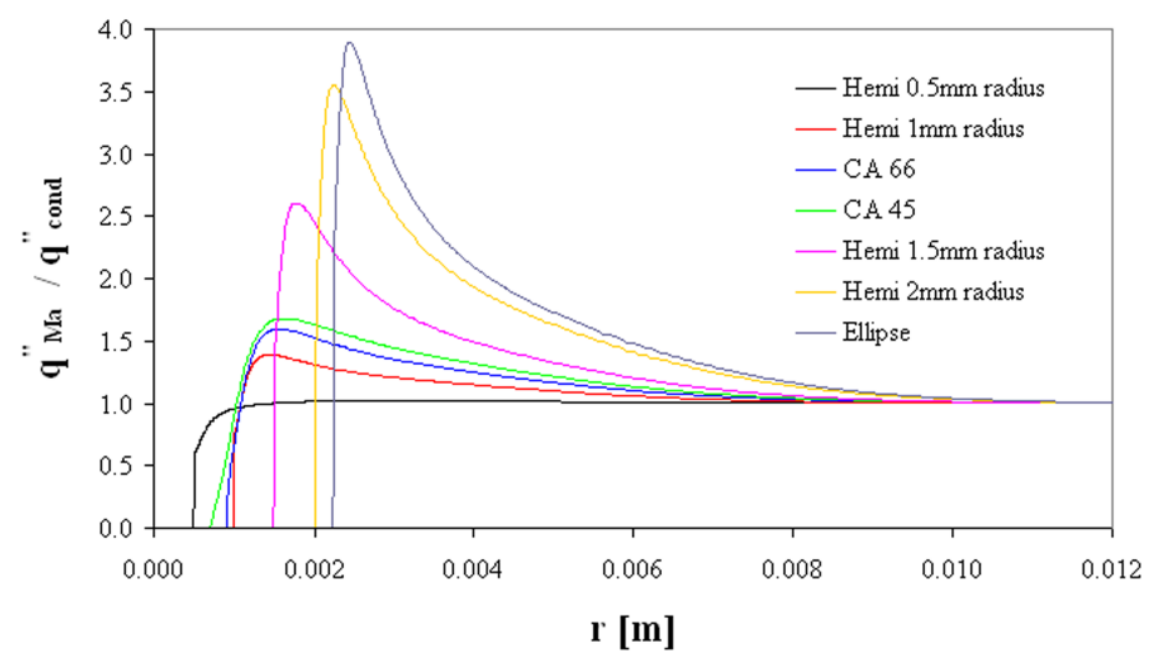

Figure 4: Local hot wall heat flux enhancement

Figure 4 plots the local heat flux enhancement along the hot wall for each of the bubble geometries simulated. Clearly, each bubble has its own degree of enhancement, but with increasing bubble size, the effective area of enhancement increases. Figure 5 plots these individual effective areas for the hot and cold walls. The $0.5 \mathrm{~mm}$ radius bubble shows no hot wall heat transfer enhancement as defined by Eq 11, since an improvement of less than 5\% was obtained. Evidently with subsequent simulations, the effective area increases with 
increasing bubble volume. There is significant difference between the $1 \mathrm{~mm}$ radius hemispherical bubble and the truncated bubbles. For the hot wall this is expected as the bubble shapes are quite different, and since the effective area is measured from the triple interface. However, a less obvious difference is noticeable on the cold wall, since the cold surface heat transfer is affected more by the intensity of the jet-like flow leaving the bubble apex. For bubbles of similar radius, this jet will affect a similar sized region of the cold wall. The cold wall results coincide behaviorally with those seen for the hot wall, albeit the effective areas are smaller for each case. Furthermore, for the $0.5 \mathrm{~mm}$ bubble, heat transfer enhancement (as defined by Eq 11) occurs at the cold wall since the jet-like flow is strong enough to impact on the cold wall.

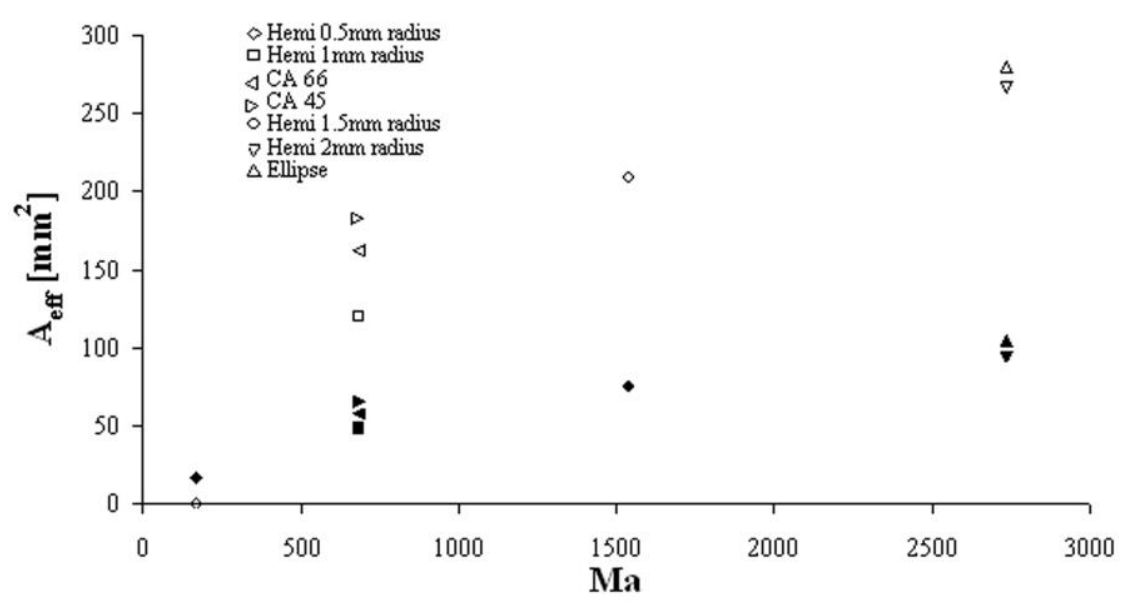

Figure 5: Effective area ( $\square$ hot wall $\square$ cold wall)

Since the bubble geometry changes between simulations, the total rate of heat transfer enhancement for this study is expressed as a Nusselt number, describing the ratio of heat transfer by Marangoni convection over the individual effective area, relative to pure conduction without a bubble over the same area.

$$
N u=\frac{Q_{M a}}{Q_{c o n d}} ; \quad N u_{h w}=\frac{\int_{r_{c p}}^{R_{e f f}} q_{M a}^{\prime \prime} 2 \pi r d r}{\int_{r_{c p p}}^{R_{e f f}} q_{c o n d}^{\prime \prime} 2 \pi r d r} ; \quad N u_{c w}=\frac{\int_{0}^{R_{e f f}} q_{M a}^{\prime \prime} 2 \pi r d r}{\int_{0}^{R_{e f f}} q_{c o n d}^{\prime \prime} 2 \pi r d r}
$$

The temperature difference between the overheated bubble base and cooler bubble tip is the driving force for Marangoni heat transfer. For the $0.5 \mathrm{~mm}$ hemispherical bubble, this temperature difference is about $2{ }^{\circ} \mathrm{C}$, whereas for the elliptical bubble, the temperature difference between bubble base and tip is about $8{ }^{\circ} \mathrm{C}$ for the same $\left(\mathrm{T}_{h}-\mathrm{T}_{\mathrm{c}}\right)$ of $50^{\circ} \mathrm{C}$. Since 
the fluid surface tension changes with temperature, and the same value of $\partial \sigma / \partial T$ is applied for each simulation, larger bubbles that protrude further into the domain will have a greater potential for Marangoni convection. This is caused by the interfacial temperature gradient. Interfacial and vortex velocities therefore increase with bubble size. The truncated bubbles extend further toward the cold wall with increasing truncation, meaning cold wall heat transfer will be affected more dramatically by bubble size than the hot wall. This behavior is depicted in Figure 6, which plots the increase in wall heat transfer along the hot and cold walls respectively. Clearly, an increase in bubble volume (therefore an increase in interfacial temperature gradient) augments local heat transfer. However, there is little disparity in the results for a $1 \mathrm{~mm}$ perfectly hemispherical bubble and the truncated bubbles of contact angle $66^{\circ}$ and $45^{\circ}$ along the hot wall. The enhancement obtained for the $2 \mathrm{~mm}$ hemispherical and bubble and the elliptical bubble also show little distinction. This leads to the conclusion that the overall hot wall heat transfer is not greatly affected by bubble shape (i.e. truncation), but rather by bubble size. Recirculation of the colder fluid towards the hot surface results in an improvement of up to $50 \%$ for an elliptical bubble. Interestingly, the results for heat transfer enhancement on the cold wall show greater response to truncation. This may be explained however, since the height of the bubble increases with truncation, meaning the cold wall is exposed to higher intensity jet-like flows leaving the apex of the bubble. The jet-like flow transports warm fluid toward the cold surface, amplifying heat transfer dramatically but over a comparatively smaller area. The combined results indicate that a hemispherical shape may not be the ideal shape for optimal heat transfer.

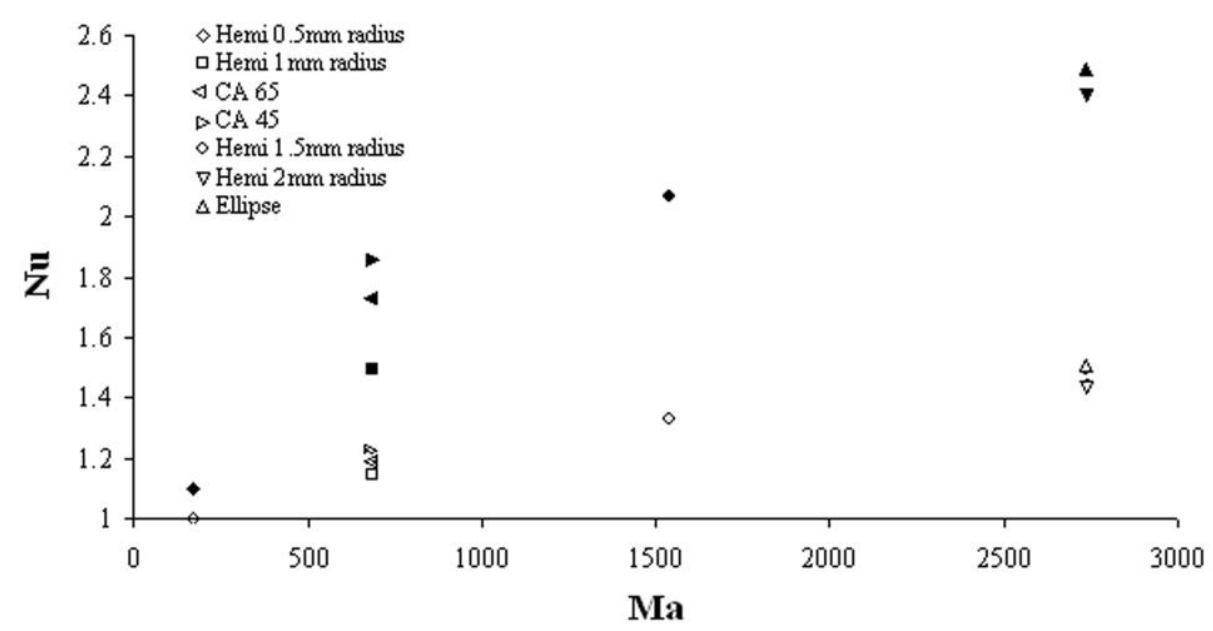

Figure 6: Heat transfer enhancement ( $\square$ hot wall $\square$ cold wall) 


\section{Conclusions}

Simulations were performed with hemispherical bubbles of different radius, as well as a truncated $1 \mathrm{~mm}$ hemispherical bubble with contact angles of $66^{\circ}$ and $45^{\circ}$ and an elliptical bubble with the same volume as a hemispherical bubble of radius $2 \mathrm{~mm}$. Increasing bubble volume causes an increase in the area over which Marangoni convection has affect. Heat transfer therefore increases with bubble size. The surface averaged hot wall heat transfer is not affected greatly by bubble shape. Both the hot and cold surface averaged heat transfer is affected dramatically by bubble size, but the increase is more profound on the cold wall. It was found that for the bubble geometries simulated, a considerable improvement in heat transfer over the effective area of up to $50 \%$ on the hot wall and $150 \%$ on the cold wall were obtained.

\section{Acknowledgements}

This work was part supported by the Irish Research Council for Science, Engineering and Technology (IRCSET) Embark Initiative.

\section{Nomenclature}

\begin{tabular}{|c|c|c|}
\hline $\mathrm{A}_{\mathrm{eff}, \mathrm{cw}}$ & effective area, cold wall & $\mathrm{m}^{2}$ \\
\hline$A_{\text {eff, hw }}$ & effective area, hot wall & $\mathrm{m}^{2}$ \\
\hline $\mathrm{CA}$ & contact angle & - \\
\hline $\mathrm{C}_{\mathrm{p}}$ & specific heat at constant pressure & $\mathrm{J} / \mathrm{kgK}$ \\
\hline $\mathrm{H}$ & liquid layer height & $\mathrm{m}$ \\
\hline $\mathrm{k}$ & thermal conductivity & $\mathrm{W} / \mathrm{mK}$ \\
\hline $\mathrm{Ma}$ & Marangoni number & - \\
\hline $\mathrm{n}$ & unit normal vector & - \\
\hline $\mathrm{Nu}$ & Nusselt number & - \\
\hline $\mathrm{Nu}_{\mathrm{cw}}$ & cold wall Nusselt number & - \\
\hline $\mathrm{Nu}_{\mathrm{hw}}$ & hot wall Nusselt number & - \\
\hline $\mathrm{p}$ & pressure & $\mathrm{N} / \mathrm{m}^{2}$ \\
\hline $\operatorname{Pr}$ & Prandtl number & - \\
\hline$q^{\prime \prime}$ & heat flux & $\mathrm{W} / \mathrm{m}^{2}$ \\
\hline
\end{tabular}




$\begin{array}{lll}\mathrm{q}^{\prime \prime} \text { cond,AB } & \text { conductive heat flux about an adiabatic bubble } & \mathrm{W} / \mathrm{m}^{2} \\ \mathrm{q}^{\prime \prime} \text { cond } & \text { conductive heat flux in the absence of a bubble } & \mathrm{W} / \mathrm{m}^{2} \\ \mathrm{Q}_{\text {cond }} & \text { conductive heat transfer in the absence of a bubble } & \mathrm{W} \\ \mathrm{q}^{\prime \prime} \mathrm{Ma} & \text { heat flux due to Marangoni convection } & \mathrm{W} / \mathrm{m}^{2} \\ \mathrm{Q}_{\mathrm{Ma}} & \text { heat transfer due to Marangoni convection } & \mathrm{W} \\ \mathrm{r} & \text { radial direction } & \mathrm{m} \\ \mathrm{R}_{\mathrm{b}} & \text { bubble radius } & \mathrm{m} \\ \mathrm{Ra} & \text { Rayleigh number } & \mathrm{m} \\ \mathrm{R}_{\mathrm{eff}} & \text { effective radius } & \mathrm{m} \\ \mathrm{r}_{\mathrm{tcp}} & \text { triple contact point location } & \mathrm{m} \\ \mathrm{T}_{\mathrm{c}} & \text { cold wall temperature } & \mathrm{K} \\ \mathrm{T}_{\mathrm{h}} & \text { hot wall temperature } & \mathrm{K} \\ \mathrm{v} & \text { velocity vector } & \mathrm{m} / \mathrm{s} \\ \mathrm{z} & \text { axial direction } & \mathrm{m}\end{array}$

\section{Greek Symbols}

$\begin{array}{lll}\alpha & \text { thermal diffusivity } & \mathrm{m}^{2} / \mathrm{s} \\ \theta & \text { azimuthal direction } & \mathrm{rad} \\ \mu & \text { dynamic viscosity } & \mathrm{Ns} / \mathrm{m}^{2} \\ \rho & \text { density } & \mathrm{kg} / \mathrm{m}^{3} \\ \sigma & \text { surface tension } & \mathrm{N} / \mathrm{m} \\ \tau & \text { shear stress vector } & \mathrm{N} / \mathrm{m}^{2} \\ v & \text { kinematic viscosity } & \mathrm{m}^{2} / \mathrm{s}\end{array}$

\section{References}

B. K. Larkin, "Thermocapillary flow around hemispherical bubble," AIChE Journal, vol. 16, pp. 101-7, 1970.

P. Arlabosse, L. Tadrist, H. Tadrist, and J. Pantaloni, "Experimental analysis of the heat transfer induced by thermocapillary convection around a bubble," Transactions of the ASME. Journal of Heat Transfer, vol. 122, pp. 66-73, 2000.

J. Betz and J. Straub, "Numerical and experimental study of the heat transfer and fluid flow by thermocapillary convection around gas bubbles," Heat and Mass Transfer/Waerme- und Stoffuebertragung, vol. 37, pp. 215-227, 2001. 
P. Arlabosse, N. Lock, M. Medale, and M. Jaeger, "Numerical investigation of thermocapillary flow around a bubble," Physics of Fluids, vol. 11, pp. 18-29, 1999.

M. Barthes, C. Reynard, R. Santini, and L. Tadrist, "Non-condensable gas influence on the Marangoni convection during a single vapour bubble growth in a subcooled liquid," Europhysics Letters, vol. 77, pp. 5 pp., 2007.

R. Raj and J. Kim, "Thermocapillary Convection during Subcooled Boiling in Reduced Gravity Environments," presented at Interdisciplinary Transport Phenomena V: Fluid, Thermal, Biological, Materials and Space Sciences, Bansko, Bulgaria, 2007.

C. Reynard, M. Barthes, R. Santini, and L. Tadrist, "Experimental study of the onset of the 3D oscillatory thermocapillary convection around a single air or vapor bubble: Influence on heat transfer," Experimental Thermal and Fluid Science, vol. 29, pp. 783-793, 2005.

S. O'Shaughnessy and A. Robinson, "Numerical investigation of bubble induced Marangoni convection," Annals of the New York Academy of Sciences, 2008. 